\title{
Baseband Version of the Bat-Inspired Spectrogram Correlation and Transformation Receiver
}

\author{
Krasin Georgiev, Alessio Balleri \\ Centre for Electronic Warfare \\ Cranfield University \\ Defence Academy of UK \\ Shrivenham, SN6 8LA, UK \\ k.georgiev@cranfield.ac.uk
}

\author{
Andy Stove \\ Stove Specialties \\ Hove, E. Sussex BN3 5NL, UK \\ andystove@compuserve.com
}

\author{
Marc Holderied \\ School of Biological Sciences \\ Bristol University \\ Bristol, BS8 1TQ, UK \\ Marc.Holderied@bristol.ac.uk
}

\begin{abstract}
Echolocating bats have evolved an excellent ability to detect and discriminate targets in highly challenging environments. They have had more than 50 million years of evolution to optimise their echolocation system with respect to their surrounding environment. Behavioural experiments have shown their exceptional ability to detect and classify targets even in highly cluttered surroundings.

The way bats process signals is not exactly the same as in radar and hence it can be useful to investigate the differences. The Spectrogram Correlation And Transformation receiver (SCAT) is an existing model of the bat auditory system that takes into account the physiology and underlying neural organisation in bats which emit chirped signals. In this paper, we propose a baseband receiver equivalent to the SCAT. This will allow biologically inspired signal processing to be applied to radar baseband signals. It will also enable further theoretical analysis of the key concepts, advantages and limitations of the "bat signal processing" for the purpose of target detection, localisation and resolution. The equivalence is demonstrated by comparing the output of the original SCAT to that of our proposed baseband version using both simulated and experimental target echoes. Results show that the baseband receiver provides compatible frequency interference pattern for two closely located scatterers.
\end{abstract}

\section{INTRODUCTION}

Recognition of a particular target amongst other interfering objects has been both a theoretical and a practical problem since the early ages of the radar systems [1]. It started with challenges like resolution of individual scatterers of a single target and dealing with complex clutter environments and advanced to cognitive sensing capabilities [2], [3]. Target image (or range profile) is implicitly associated in the literature with the reflected signal matched filtered with the transmitted signal. Matched filter theory explains the optimum predetection processing in case of white noise [4].

Behavioral experiments have demonstrated that bats are dealing successfully with the above challenges. Some species chase insects in open air but others forage for fruit or flowers in the clutter of trees and vegetation. They can use echolocation alone to detect, localise and discriminate both moving and static targets [5], [6]. Bats exploit spatial and temporal adaptations in the process of target recognition. Adaptive waveform diversity [7], cognitive guidance and control [7], [8] and memory [8], [9] are characteristics that are studied in the context of cognitive sensing. Work by radar and sonar engineers on target classification inspired by what is known about how bats perform this task has generally still assumed that the receiver includes a matched filter, e.g. see [6], [7].

Previous experiments have shown that bats are able to resolve closely located scatterers. In [10], [11], Simmons showed that the big brown bat (Eptesicus fuscus) can perceive the arrival-time of virtual echoes with an accuracy of 10-15 ns in quiet ambient laboratory conditions and of $40 \mathrm{~ns}$ for Signalto-Noise Ratio of $36 \mathrm{~dB}$. A two-point resolution is $2-10 \mu \mathrm{s}$ with waveforms of a bandwidth $85 \mathrm{kHz}$. Schmidt showed that the bat Megaderma lyra is able to discriminate between two phantom targets producing echoes with a delay between them of about $1 \mu \mathrm{s}$ [12] with waveforms of a bandwidth of up to $100 \mathrm{kHz}$, suggesting the bat capability to achieve higher range resolution performance with respect to the conventional matched filter (above $10 \mu \mathrm{s}$ or $1.7 \mathrm{~mm}$ for speed of sound $340 \mathrm{~m} / \mathrm{s})$. Discrimination of surface structures is demonstrated in [13] where Glossophaga soricina were trained to distinguish smooth surface from coarse with a structure depth bigger than $0.38 \mathrm{~mm}$. The emitted frequency modulated down-chirp had three harmonics - 95-55 kHz, $150-86 \mathrm{kHz}$ and 190 $140 \mathrm{kHz}$. This is one of the few experiments with real targets, demonstrating fine resolution capabilities compatible to the ones with phantom targets.

The sensitivity of bats to disruption of the echo spectrograms was examined as an explanation of the clutter suppression mechanism [14]. By introducing different delays to the echo harmonics it was shown that misalignments as small as $2.6 \mu \mathrm{s}$ can degrade echo delay discrimination significantly. This effect is described as "defocusing" of the bat's delay image. One hypothesis is that the frequency interference pattern introduced by closely spaced targets is converted into temporal misalignments in the auditory image. This effect combined with different attenuations at different frequencies leads to defocusing of the clutter [14]-[16]. An important role in this process is attributed to the harmonic structure of the signal and the lower width of the sonar beam at higher frequencies (leading to attenuation of the higher frequencies for returns from targets not centred in the sonar beam). All experiments discussed above were performed with stationary targets and Doppler cues were not available to the bat. 
Different aspects of the way bats process echoes from targets and the surrounding environment are studied in the literature. These models [17], [18], [19] use only range information, a single receiver, and do not account for directional sensitivity. In these models frequency modulated signals (emitted call and received echoes) are passed through a filter bank to generate auditory spectrogram (a model for the cochlea) which is followed by temporal processing (a model for delay tuned neurons in the brain) for ranging the targets and a spectral processing to resolve close target interferences [17], [19].

The Spectrogram Correlation and Transformation (SCAT) receiver is a model of the auditory system that was proposed by Saillant et al. in [17] for the bat Eptesicus fuscus. It accounts for the underlying neural organisation in FM bats by special emphasis on the physiological correlation of the model. The main modules of the SCAT are the cochlear block, the temporal (spectrogram correlation) block and the spectral (spectrogram transformation) block. A detailed analytical modelling of the temporal block was presented by Peremans and Hallam [20]. The closed-form equations approximating the spectral block are derived by Park and Allen [21]. In these analysis of SCAT the outputs of the bandpass filters were approximated with cosines shaped by Gaussian envelopes.

While the aim of the bat auditory system models is to reproduce the acoustic images perceived by bats in a variety of behavioural experiments, there is no explanation on what exactly brings the performance improvement so it could be exploited in a technological system. Bat precision in echo resolution is presumed by some authors to require a fully coherent receiver so these authors try to preserve the phase of the sound wave in their models to implement coherent signal processing [17], [18], [20]. Others argue that a coherent receiver is physiologically implausible [19]. The effect of bandpassed signal smoothing on coherence is explored in [22].

In this paper we propose a baseband receiver equivalent to the SCAT [17]. It is denoted as BSCT (Baseband Spectrogram Correlation and Transformation). The equivalence of BSCT to SCAT will be demonstrated by comparing the model output of the original and baseband model using both simulated signals and laboratory measurements.

Our baseband model will allow the processing of target echoes with a receiver based on the bat auditory system even for signals that are centred on very high carrier frequencies, such as radar signals. It has the advantage to significantly simplify the analytical treatment of such receivers and hence can be used for further investigations. This will be the foundation for further study with the ultimate goal to understand how bat signal processing differs from matched filtering and explain its advantages for the task of target identification.

The scope of the study presented here is limited to the spectral part of the model. The spectral block is important because it provides the processing algorithm and fine resolution image output needed for target discrimination [17]. Its output is different from the conventional matched filter output.

The results are compared by calculating the differences between both model outputs for different experimental settings.
The results show that the root mean square error is less than $1 \%$ for delays below $50 \mu$ s and less than $5 \%$ up to $100 \mu \mathrm{s}$. This is valid both for simulations and experiments.

\section{DESCRIPTION OF THE SPECTROGRAM CORRELATION AND TRANSFORMATION MODEL}

The bat auditory system processes both the emitted call and the received echo through an auditory periphery and then through some higher level brain structures. The auditory periphery includes the outer, the middle and the inner ear and provides a time-frequency representation of the input signal. The frequency content is sampled in a non-linear scale (hyperbolic or logarithmic). The consequent brain processing provides an estimate of the time delay between the call and the echo and provides cues about the structure of each echo.

A short summary of the SCAT model building blocks based on [17] follows. Notes on alternative models are also included.

\section{A. Cochlear block}

The cochlear block is modelled with a bank of 81 Butterworth band-pass filters of order 10 and bandwidth $B=4 \mathrm{kHz}$. Each filter is followed by a signal rectifier and a $3 \mathrm{kHz}$ bandwidth low-pass filter (Fig. 1) in order to extract the envelope of the signal.

The central frequencies $f_{c}$ of the band-pass filters span the bandwidth between $20 \mathrm{kHz}$ to $100 \mathrm{kHz}$ and are arranged in a hyperbolic scale as $f_{c}=1 / T_{c}$, where the central period $T_{c}$ changes linearly from $10 \mu \mathrm{s}$ to $50 \mu \mathrm{s}$ with increments of $0.5 \mu \mathrm{s}$.

Two levels of smoothing of the envelope have been proposed with the SCAT, namely a high smoothing and a low smoothing with the high smoothing consisting of a full wave rectifier followed by a second order low-pass filter, whilst the low smoothing consists of a half wave rectifier followed by first order low-pass filter.

In both cases the low-pass filter is a IIR Butterworth with a bandwidth of $3 \mathrm{kHz}$.

The output of the cochlea is called auditory spectrogram or cochleogram (Fig. 4).

In the literature, some modified versions of the original SCAT have been proposed which differ on how the initial splitting of the signal into frequency channels is carried out. For example the bank of constant bandwidth Butterworth filters is replaced by gammatone filters [19] with frequency dependent bandwidth after [23] or Gaussian chirplets with carrier frequencies compatible with the emission sweep rate [18]. Some models use additional non-linear transformation, e.g. raising the amplitude of the detected signal to a power of 0.4 to account for the non-linear interactions in the organ of corti [19].

\section{B. Temporal block}

The purpose of the temporal block is to estimate the time delay between the call and the echo based on the output of the cochlear block. It consists of a set of tapped delay lines 


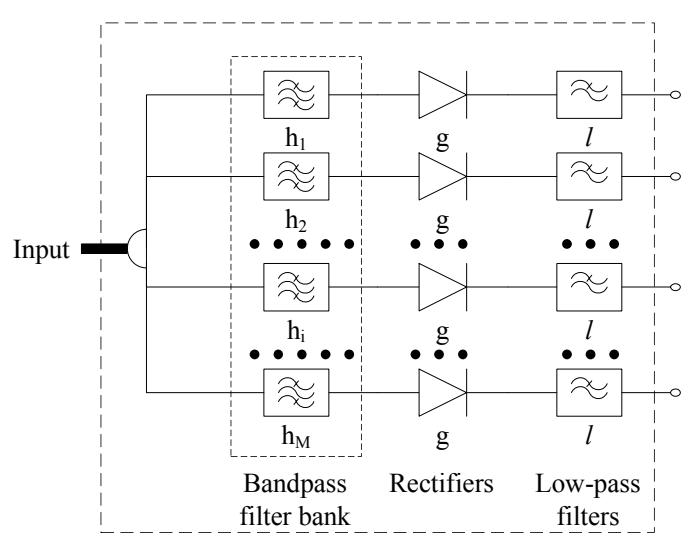

Fig. 1. Cochlear block of SCAT receiver. Filter bank of $M=81$ bandpass filters $h_{i}$ with central frequencies from $20 \mathrm{kHz}$ to $100 \mathrm{kHz}$. Rectifier $g$ and Butterworth low-pass filter $l$ follow after each bandpass filter

that implement a cross-correlation function between the call and the echo. These are triggered by the call signal.

The temporal block processes the output of the cochlear block and converts it into neural spikes. The neural coding can either be considered as part of the cochlear block or not. The spike decay period is $4 \mu \mathrm{s}$ [17]. A spike rise results in an increase of the threshold and inhibits the same neuron to produce another spike. The spike activation threshold decays to its initial value over a period of $1 \mathrm{~ms}$.

The temporal block carries out a "dechirping" of the signal by adding appropriate delays to each frequency channel (Fig. 5). Delays are calculated using the emitted signal as a trigger. Simultaneous activity in multiple channels is detected by a set of coincidence detection neurons and is a sign of the target presence. Target detection is implemented by summing the output over all channels and the target is declared with a peakdetection algorithm.

\section{Spectral block}

The spectral block is responsible for extracting the fine structure of the target. It is used to detect and measure the delay between highly overlapping echoes, which cannot be resolved by the temporal block.

The spectral block exploits the interference pattern between overlapping echoes, which results in the suppression or amplification of the power of the output of some of the filters of the cochlear block. It integrates the output signal of each frequency channel for a specific time interval (about $350 \mu \mathrm{s}$ [17]). De-chirp delays are also compensated so that only the signals coming from the same target are integrated.

The output of the integration represents the frequency spectrum of the signal. A modified inverse cosine transform is then used to convert the frequency spectrum back into the time domain. Some studies concentrate on time domain reconstruction of the target image [17], [18], [20] but others emphasise the spectral based perception of the target [13], [19]. A "pattern-matching" interpretation of the transformation of spectral interference patterns into fine delays is proposed in [21].

\section{Model output}

The outputs of temporal and spectral blocks could be considered separately. For a group of closely positioned targets the former gives information about location on the time (range) axis for the group as a whole, and the later describes the intragroup behaviour either as spectrum reflecting the interferences or as fine delays (relative distances).

\section{BASEBAND EQUiVALENT OF THE SPECTROGRAM TRANSFORMATION}

The baseband equivalent of the spectrogram transformation proposed in this paper deals with analytical input signal. Let us have a filter bank composed of $M$ filters with central frequencies $f_{i}, i=0 \ldots M-1$, bandwidth $B_{i}$ and all having the same shape. Assuming that all filters are with the same bandwidth $B$, the impulse response $h_{i}(f)$ of any filter $i$ in the filter bank can be produced from the same baseband (low-pass) filter with impulse response $h(f)$ by spectral shift:

$$
h_{i}(t)=h(t) e^{j 2 \pi f_{i} t}
$$

The response at the output of the $i$ 'th filter to the signal $x(t)$ is the convolution of the filter impulse response and the signal:

$$
y_{i}(t)=x(t) * h_{i}(t)
$$

After each bandpass filter the cochlear block contains a rectifier and a low-pass filter (Fig. 1). We replace them with an ideal amplitude extractor. Therefore the envelope of the bandpass filtered signal is produced by taking the amplitude of the signal (Fig. 4). This alternative to the signal rectification and low-pass filtering discards completely the phase information. Then the envelopes are converted to an auditory spectrogram by squaring (Fig. 5).

In the spectral block the total energy of the output of each filter is computed by integration (Fig. 2). In order to separate the spectral signature of multiple groups of scatterers, integration is limited to an interval around the location of the group under consideration (Fig. 5). This way the spectrum is calculated for each group. Group locations for each filter are produced by the temporal block.

In case of a single group of scatterers the spectral block can be simplified by ignoring the output of the temporal block and integrating the whole filter output. The spectral output of BSCT can be written:

$$
E[i]=\int_{-\infty}^{\infty}\left|y_{i}(t)\right|^{2} d t
$$

Finally the spectral output (Fig. 6) can be inverse transformed into time (space) so both temporal and spectral output can be presented on the delay (range) axis. 


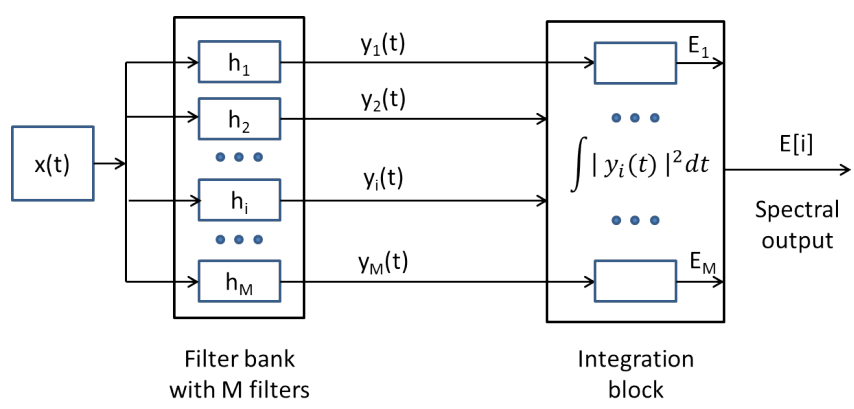

Fig. 2. BSCT spectral processing diagram. Model input $x(t)$ is analytical signal. It is passed through filter bank of $\mathrm{M}$ complex bandpass filters $h_{i}$. The absolute value of each filter output $y_{i}$ is squared and integrated over time to get the energy $E[i]$ of the corresponding frequency.

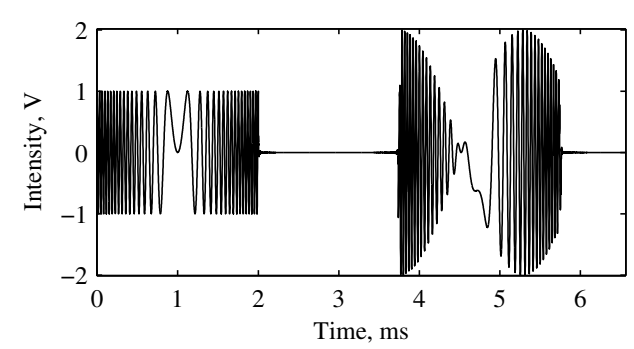

Fig. 3. Baseband model input, real component. The signal frequency is shifted to baseband. Emitted call has duration $2 \mu$ s and spans linearly from $-32.5 \mathrm{kHz}$ to $+32.5 \mathrm{kHz}$. Received echo comes from two scatterers with delay $20 \mu$ s and equal amplitude.

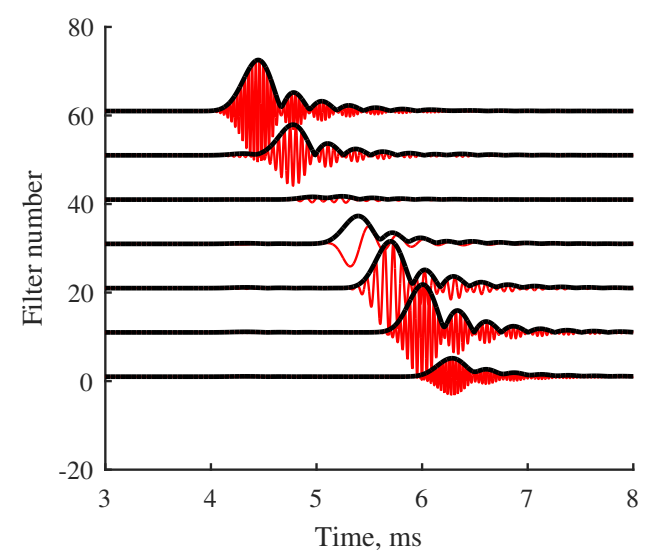

Fig. 4. Bandpass filter bank output, in-phase (real) component and envelope

\section{EXPERIMENT SETUP}

Data collection includes both simulations and real experiments for a set of targets with the same parameters. Both the simulated and the measured data have been processed using both the original SCAT algorithm and our BSCT. The comparison between the two algorithms using simulated data can be expected to be successful since the behaviour of the algorithms can be predicted in advance, but this is a useful baseline for the comparison of how the two algorithms behave with real data with its practical imperfections.

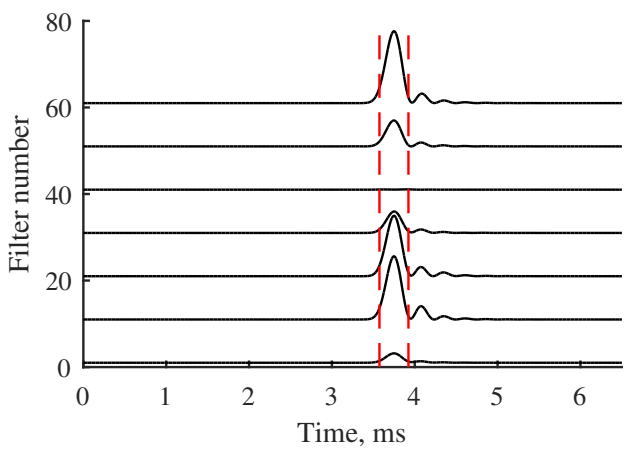

Fig. 5. Example of de-chirped squared envelopes for seven filters and integration interval (red dashed lines) over which the total energy for each filter is calculated

\section{A. Data collection}

a) Digitization: All signals were sampled at $1 \mathrm{MHz}$ rate. In total 10000 samples were saved per measurement, which corresponds to signal duration of $10 \mathrm{~ms}$. Digital to analogue and analogue to digital conversion were done with TiePie Handyscope HS5-540 dual channel oscilloscope with function generator.

b) Emitted signal: The transmission (or "bat call", $x_{C}(t)$ ) was a linear chirp from $100 \mathrm{kHz}$ down to $35 \mathrm{kHz}$ with duration of $2 \mathrm{~ms}$. The same call signal is used for both the simulations and for the experiments.

c) Targets and echo: Two close-spaced point targets were created by producing two time delayed versions of the emitted calls. The relative position of the targets was varied by using different values for the delay $\tau[5,10,15,20,25,50,100]$ $\mu s$, corresponding to separations of $[0.85,1.7,2.55,3.4,4.25$, $8.5,17] \mathrm{mm}$. The impulse response of the targets will be:

$$
x_{T}(t)=\delta(t)+\delta(t-\tau)
$$

where $\delta(t)$ is the Dirac delta function.

The received signal (or "echo"), $x_{E}(t)$ is the reflection of the call signal from the target. For the simulations it was generated by time shifting the call by $t_{1}=3740 \mu \mathrm{s}$ and convolving with the target:

$$
x_{E}(t)=x_{C}\left(t-t_{1}\right) * x_{T}(t)
$$

The real measurements were recorded with an ultrasound microphone (type CM16, Avisoft Bioacoustics, Berlin, Germany). A phantom target was created using an ultrasound loudspeaker (type S55/6, Ultra Sound Advice, London, UK). Reflection from two scatterers was reproduced by emitting not just the call $x_{C}(t)$ but the call convolved with the target $x_{C}(t) * x_{T}(t)$. The delay between the call and the first target $t_{1}$ was achieved by putting the speaker at distance $1.272 \mathrm{~m}$ from the microphone.

Both simulations and measurements reproduce reflections from the same targets - two ideal point targets with the specific separations. The measurements however include the effect of air attenuation, noise and transmitter/receiver imperfections. 
TABLE I

DIFFERENCE BETWEEN PROPOSED BSCT AND ORIGINAL SCAT WITH HIGH (SCAT-H) AND LOW (SCAT-L) SMOOTHING, EXPESSED AS RMS, \%

\begin{tabular}{cccccc}
\hline \multirow{2}{*}{$\begin{array}{c}\text { Delay } \\
\mu \mathrm{s}\end{array}$} & \multicolumn{2}{c}{ Simulations, \% } & & \multicolumn{2}{c}{ Experiments, \% } \\
\cline { 2 - 3 } \cline { 5 - 6 } & SCAT-H & SCAT-L & & SCAT-H & SCAT-L \\
\hline 5 & 0.20 & 0.19 & & 0.78 & 0.81 \\
10 & 0.54 & 0.68 & & 0.39 & 0.42 \\
15 & 0.34 & 0.34 & & 0.38 & 0.39 \\
20 & 0.67 & 0.52 & & 0.60 & 0.59 \\
25 & 0.41 & 0.39 & & 0.57 & 0.55 \\
50 & 0.94 & 0.98 & & 0.85 & 0.90 \\
100 & 4.4 & 4.6 & & 2.1 & 2.1 \\
\hline
\end{tabular}

\section{B. Data processing}

All experimental measurements were preprocessed by removing the mean value.

The inputs to BSCT were converted to baseband by multiplying the Hilbert transform with the carrier (Fig. 3).

Both SCAT and BSCT were implemented in Matlab (release 2015a, MathWorks, Natick Mass., USA). A linear frequency spacing of the filters in the bandpass filter bank is used to allow processing of a linear chirp. The filter bank contains 65 bandpass filters with linear frequency spacing from -32 to 32 $\mathrm{kHz}$ for BSCT and from 35.5 to $99.5 \mathrm{kHz}$ for SCAT $(1 \mathrm{kHz}$ increments). All other parameters follow the ones described in sec. II after [17]. Two versions of the SCAT differing in the level of smoothing are considered labelled SCAT-L for low smoothing and SCAT-H for high smoothing.

Finally the output of the spectral block for each model is calculated by passing the call and echo signals through the model and normalising by dividing by the maximum value of each output.

\section{REsults}

The spectral output of the proposed BSCT is compared with the output of both versions of the original SCAT (with low and high smoothing) for different delays between the glints in the target. The difference is measured by using the root mean square (RMS) error:

$$
\text { error }=\sqrt{\frac{1}{M} \sum_{i=1}^{M}\left(E_{S C A T}\left[f_{i}\right]-E_{B S C T}\left[f_{i}\right]\right)^{2}}
$$

where $E_{S C A T}$ and $E_{B S C T}$ are the normalised outputs of the models compared and $M=65$ is the number of filters.

The results of the comparison expressed as RMS error in $\%$ of maximal value are presented in Table I for all datasets. Results show the error is less than $1 \%$ for delays below $50 \mu \mathrm{s}$ and less than $5 \%$ up to $100 \mu \mathrm{s}$. Fig. 6 displays the full outputs of BSCT and SCAT expressed as a function of the frequency for the case of two simulated targets delayed by $20 \mu \mathrm{s}$. We can see that the level of smoothing in the original SCAT does not influence the spectral output. The same is valid for the baseband model which gives practically the same results.
TABLE II

ROOT MEAN SQUARED DIFFERENCE BETWEEN SIMULATIONS AND EXPERIMENTS PROCESSED WITH BSCT

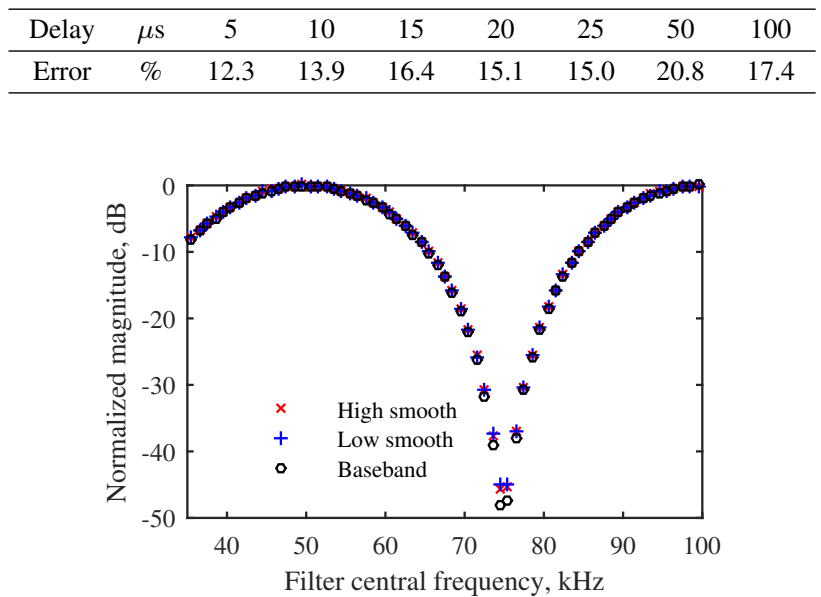

Fig. 6. Spectral output of BSCT (baseband) and SCAT (high and low smoothing), simulated two targets with separation $3.4 \mathrm{~mm}$ (delay $20 \mu \mathrm{s}$ )

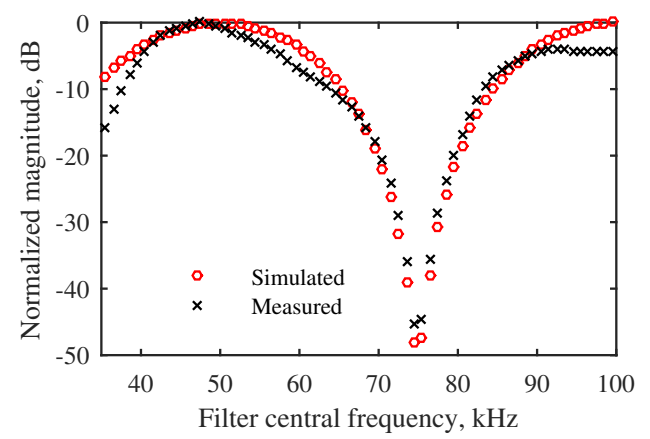

Fig. 7. Spectral output of BSCT model, simulated vs measured two targets separated by $3.4 \mathrm{~mm}$ (delay $20 \mu \mathrm{s}$ )

In the real measurements, the influence of the air and non-perfect microphone and speaker characteristics modify the target response. The outputs of BSCT for simulated and phantom targets are compared in Table II and shown in Fig. 7. The real experiments introduce significant deformation of the target spectrum relative to the simulations - difference above $10 \%$. This shows that although the real data behaves in a way which is not identical to the simulated data - as would be expected, the two algorithms are stable to the imperfections in the data and both still behave in a similar manner.

Looking closer to the interference patterns for different relative positions between the scatterers (Fig. 8) we can see that the general shape and, in particular, the locations of the zeroes, are preserved between the experiments and the simulations. These features are likely to be significant for any scheme for resolving the close-spaced targets and this result indicates that the new algorithm, like the SCAT algorithm, retains the information which will later be needed to resolve the targets. 


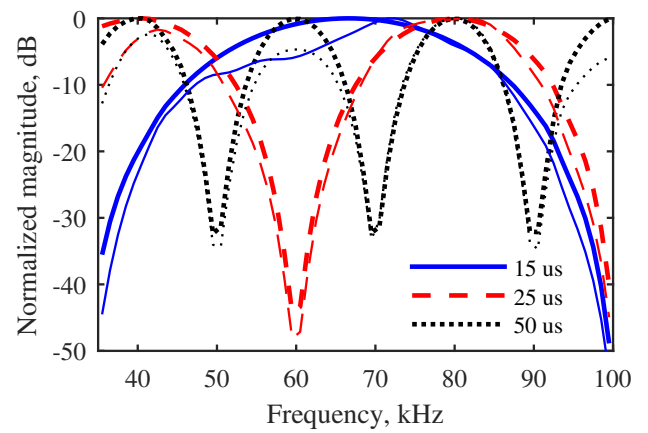

Fig. 8. Spectral output of BSCT model for two targets for delays $15 \mu \mathrm{s}, 25 \mu \mathrm{s}$ and $50 \mu \mathrm{s}$. Simulated (thick lines) and measured (thin lines) input signals

\section{CONCLUSION}

The proposed baseband spectrogram transformation model gives an output compatible with the output of the original spectrogram correlation and transformation receiver. This implies that

- processing of target echoes with a receiver based on the bat auditory system can be applied to signals that are centred on very high carrier frequencies, such as radar signals.

- the output of the spectral block does not depend on the phase information of the carrier signal and is a form of non-coherent signal processing; the spectral block will be more robust to loss of signal coherence than the matched filter.

- advanced signal analysis techniques based on complex signal representation could be used for further understanding of the model.

\section{ACKNOWLEDGMENT}

Krasin Georgiev and Alessio Balleri thank Cranfield Defence and Security for funding their work under the CDS PhD bursary scheme.

\section{REFERENCES}

[1] A. W. Rihaczek, Principles of high-resolution radar. New York: McGraw-Hill, 1969.

[2] D. Blacknell and H. Griffiths, Eds., Radar Automatic Target Recognition (ATR) and Non-Cooperative Target Recognition (NCTR). Institution of Engineering and Technology, 2013.

[3] G. W. Stimson, H. D. Griffiths, C. J. Baker, and D. Adamy, Stimson's Introduction to Airborne Radar (3rd Edition). Institution of Engineering and Technology, 2014.

[4] C. E. Cook and M. Bernfeld, Radar Signals: An Introduction to Theory and Application. New York: Academic Press, 1967.

[5] D. von Helversen, "Object classification by echolocation in nectar feeding bats: size-independent generalization of shape." Journal of comparative physiology. A, Neuroethology, sensory, neural, and behavioral physiology, vol. 190, no. 7, pp. 515-21, 72004.

[6] A. Balleri, H. D. Griffiths, C. J. Baker, K. Woodbridge, and M. W. Holderied, "Analysis of acoustic echoes from a bat-pollinated plant species: Insight into strategies for radar and sonar target classification," IET Radar, Sonar and Navigation, vol. 6, no. 6, pp. 536-544, 2012.

[7] C. J. Baker, H. D. Griffiths, and A. Balleri, "Biologically inspired waveform diversity," in Waveform Design and Diversity for Advanced Radar Systems, ser. Radar, Sonar, Navigation and Avionics, F. Gini, A. De Maio, and L. Patton, Eds. Institution of Engineering and Technology, 2012, vol. 22, ch. 6, pp. 149-172.
[8] C. J. Baker, G. E. Smith, A. Balleri, M. Holderied, and H. D. Griffiths, "Biomimetic echolocation with application to radar and sonar sensing," Proceedings of the IEEE, vol. 102, no. 4, pp. 447-458, 2014.

[9] J. R. Barchi, J. M. Knowles, and J. A. Simmons, "Spatial memory and stereotypy of flight paths by big brown bats in cluttered surroundings," Journal of Experimental Biology, vol. 216, no. 6, pp. 1053-1063, 2013.

[10] J. A. Simmons, M. Ferragamo, C. F. Moss, S. B. Stevenson, and R. A. Altes, "Discrimination of jittered sonar echoes by the echolocating bat, eptesicus fuscus: The shape of target images in echolocation," Journal of Comparative Physiology A, vol. 167, no. 5, pp. 589-616, 111990.

[11] J. A. Simmons, P. A. Saillant, J. M. Wotton, T. Haresign, M. J. Ferragamo, and C. F. Moss, "Composition of biosonar images for target recognition by echolocating bats," Neural Networks, vol. 8, no. 7-8, pp. 1239-1261, 1995.

[12] S. Schmidt, "Perception of structured phantom targets in the echolocating bat, megadermalyra," The Journal of the Acoustical Society of America, vol. 91, no. 4, pp. 2203-2223, 1992.

[13] R. Simon, M. Knörnschild, M. Tschapka, A. Schneider, N. Passauer, E. K. V. Kalko, and O. von Helversen, "Biosonar resolving power: echoacoustic perception of surface structures in the submillimeter range." Frontiers in physiology, vol. 5, p. 64, 12014.

[14] M. E. Bates and J. a. Simmons, "Perception of echo delay is disrupted by small temporal misalignment of echo harmonics in bat sonar," The Journal of experimental biology, vol. 214, no. Pt 3, pp. 394-401, 22011.

[15] J. A. Simmons and J. E. Gaudette, "Biosonar echo processing by frequency-modulated bats," IET Radar, Sonar and Navigation, vol. 6, no. 6 , pp. 556-565, 2012.

[16] M. E. Bates, J. A. Simmons, and T. V. Zorikov, "Bats use echo harmonic structure to distinguish their targets from background clutter." Science (New York, N.Y.), vol. 333, no. 6042, pp. 627-30, 72011.

[17] P. A. Saillant, J. A. Simmons, S. P. Dear, and T. A. McMullen, "A computational model of echo processing and acoustic imaging in frequency-modulated echolocating bats: The spectrogram correlation and transformation receiver," The Journal of the Acoustical Society of America, vol. 94, no. 5, pp. 2691-2712, 111993.

[18] I. Matsuo, K. Kunugiyama, and M. Yano, "An echolocation model for range discrimination of multiple closely spaced objects: transformation of spectrogram into the reflected intensity distribution," The Journal of the Acoustical Society of America, vol. 115, no. 2, pp. 920-928, 22004.

[19] L. Wiegrebe, "An autocorrelation model of bat sonar," Biological cybernetics, vol. 98, no. 6, pp. 587-595, 2008.

[20] H. Peremans and J. Hallam, "The spectrogram correlation and transformation receiver, revisited," The Journal of the Acoustical Society of America, vol. 104, no. 2, pp. 1101-1110, 81998.

[21] M. Park and R. Allen, "Pattern-matching analysis of fine echo delays by the spectrogram correlation and transformation receiver," The Journal of the Acoustical Society of America, vol. 128, no. 3, pp. 1490-1500, 2010.

[22] M. I. Sanderson, N. Neretti, N. Intrator, and J. A. Simmons, "Evaluation of an auditory model for echo delay accuracy in wideband biosonar." The Journal of the Acoustical Society of America, vol. 114, no. 3, pp. 1648-59, 92003.

[23] R. D. Patterson, M. H. Allerhand, and C. Giguère, "Time-domain modeling of peripheral auditory processing: a modular architecture and a software platform." The Journal of the Acoustical Society of America, vol. 98, no. 4, pp. 1890-4, 101995. 